\title{
Anesthesic Management of Cervical Disc Herniation During Prégnancy: A Case Report
}

Mehdi Samali (MD) ${ }^{*}$, Mohammed Rabi Andaloussi (MD), Said Khallikane (MD), Amine Meskine (MD), Abdelghafour Koundi (MD), Mustapha Bensghir (PhD)

Department of Anesthesiology and Intensive Care, Military Hospital Mohammed V Rabat, Faculty of Medicine and Pharmacy of Rabat, University Mohammed V Rabat, Morocco

DOI: $10.36347 /$ simcr.2020.v08i10.001

| Received: 21.09.2020 | Accepted: 29.09.2020 | Published: 03.10.2020

*Corresponding author: Mehdi Samali

Abstract

Cervical herniation during pregnancy is a extremely rare event requiring emergent decompressive surgery in the majority of cases to prevent permanent neurological damage. Therefore, there is little data in the literature regarding anesthetic management of cervical localization during pregnancy. The potential for difficult airway management with the patient under general anesthesia is one of the major concerns that needs to be addressed to prevent further cord compression. Anesthesic management should also include measures to maintain the mean arterial pressure to improve spinal cord perfusion. Furthermore, spine surgery in pregnant patients needs special consideration in terms of positioning and in the postoperative period. We present a case of a 28 -year-old white woman at 18 weeks of gestation with a cervical disc herniation. Fiberoptic bronchoscope-guided nasal intubation was a safe option to ensure a higher rate of successful endotracheal intubation while minimizing the risk of aggravating the injury. Her care posed other multiples challenges that required a multidisciplinary team approach. Conclusions: The case of our patient serves as a reminder of this rare condition and its implications regarding anesthesia.

Keywords: Cervical, disc, herniation, Pregnancy, Anesthesic, Fiberoptic bronchoscope intubation.

Copyright @ 2020: This is an open-access article distributed under the terms of the Creative Commons Attribution license which permits unrestricted use, distribution, and reproduction in any medium for non-commercial use (NonCommercial, or CC-BY-NC) provided the original author and source are credited.

\section{INTRODUCTION}

Cervical disc herniation $(\mathrm{CDH})$ during prégnancy is extremely rare. The aim of this case report was to present an anesthetic approach in surgery performed in a prégnant women at 18 weeks of gestation with an $\mathrm{CDH}$.

\section{Case Presentation}

A 28 -year-old white woman (weight $78 \mathrm{~kg}$, height $175 \mathrm{~cm}$ ), gravida 2 para 1 , presented to our hospital at 18 weeks of gestation with paraparesis. She had left cervico brachial neuralgia and no anesthetic history; in particular, she had no history of trauma. She had been receiving regular care from an obstetrician during pregnancy, which had progressed normally. 2 days before her admission, the patient had developed a sudden onset of severe interscapular pain radiating to her neck with sudden weakness and paresthesia in both the upper and lower limbs. after 24 hours no improvement was observed, so she decided to consult in our hospital.Her physical examination revealed a paraparesis in both legs. Her lower limb power bilaterally was grade $2 / 5$. In her right and left upper limbs, power was grades $2 / 5$ and $3 / 5$, respectively. Her upper level with sensitivity was C5-C6. Her plantar response was bilaterally mute. She had urinary sphincter disturbances. All her deep tendon reflexes were absent.The rest of her physical examination was unremarkable.The results of hematological investigations were within normal ranges: serum potassium 3,8 $\mathrm{mEq} / \mathrm{L}$, hemoglobin $15 \mathrm{~g} / \mathrm{dl}$, platelets $165,000 / \mathrm{mm} 3$, international normalizedratio 1.1 , and prothrombin time 11 seconds. Obstetrical ultrasound revealed a normal pregnancy at 18 weeks of gestation and a fetal heart rate (HR) of 140 beats per minute. Magnetic resonance imaging (MRI) of the cervical and dorsal spine revealed a cervical spine disc herniation C5-C6 with severe spinal cord compression (Fig-1). 

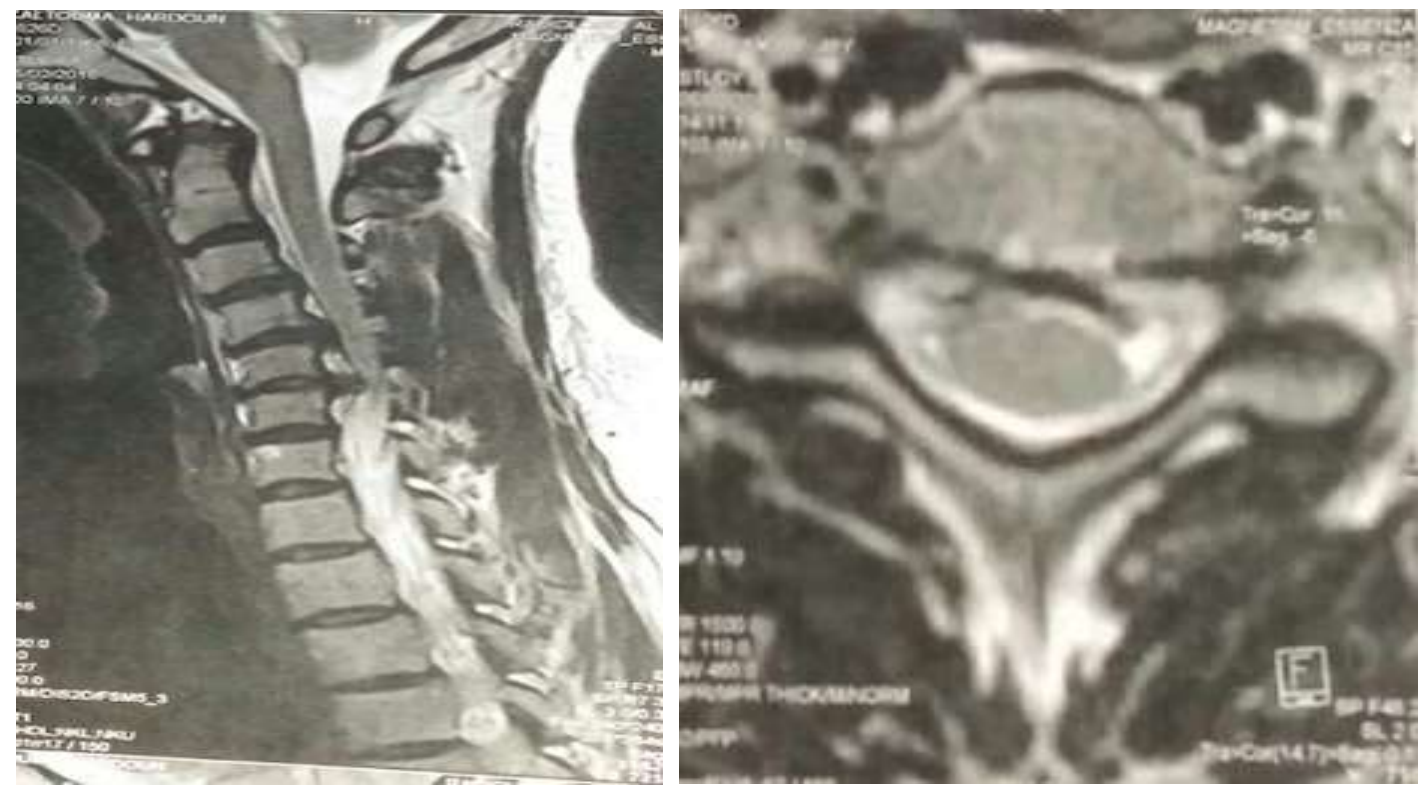

Fig-1: Sagital T2 (a) and axial (b) MRI sequence showing adjacent to C5-C6 a left paramedian disc protrusion exerting a mass effect on the cervical spinal cord

An anesthesiologist, a neurosurgeon, and an obstetrician urgently reviewed the case. After discussion with the patient, it was decided to perform spinal cord decompression with the patient under general anesthesia.

After explanation of the anesthesia plan, the patient verbalized understanding and consented to undergo awake fiberoptic intubation. Her American Society of Anesthesiologists Physical Status classification was I, her Mallampati classification was I, and she had a good mouth opening. On arrival in our operating room (3 hours from admission), non invasive blood pressure (BP) monitoring with five-lead electrocardiography and pulse oximetry was established. The patient's basal values were BP 130/70 $\mathrm{mmHg}, \mathrm{HR} 70$ beats per minute and peripheral capillary oxygen saturation of $99 \%$ while breathing room air.

Intravenous access was secured with two 18gauge intravenous cannulae, and the patient was given $10 \mathrm{ml} / \mathrm{kg}$ of isotonic saline solution. Cefazolin $2 \mathrm{~g}$ was also administered intravenously. After 5 minutes of preoxygenation with $100 \%$ oxygen and nebulization of lidocaine $2 \%$, we carried out a superior laryngeal block, which was followed by nasal fiberoptic intubation. Tracheal intubation was easily performed with a 7.0$\mathrm{mm}$ armored tube. Once the patient's end-tidal carbon dioxide level confirmed placement, general anesthesia was induced with propofol $2.5 \mathrm{mg} / \mathrm{kg}$, fentanyl 3,5 $\mu \mathrm{g} / \mathrm{kg}$, and rocuronium $0.6 \mathrm{mg} / \mathrm{kg}$. Immediately after intubation, the patient's BP was $120 / 65 \mathrm{mmHg}$, and her HR was 85 beat per minute. She was ventilated with a target tidal volume of $480 \mathrm{ml}$ and a respiratory rate of 12 breaths per minute. Sevoflurane $(1-1.5 \%)$ in a mixture of oxygen and air (1:2) was used for maintenance of anesthesia. A radial arterial line was placed for continuous monitoring of arterial BP and collection of arterial blood for blood gas analysis during surgery. We also inserted a urinary catheter.

The patient was maintained in the supine position to allow a anterior cervical approach to the disc herniation. The endotracheal tube was rechecked for correct position by chest auscultation. The patient's airway pressure was $24 \mathrm{cmH} 2 \mathrm{O}$. Intraoperative fetal monitoring was accomplished. The patient's mean BP was maintained between 85 and $95 \mathrm{mmHg}$. Perioperatively, blood gas analysis showed $\mathrm{pH} 7.42$, partial pressure of carbon dioxide $37 \mathrm{mmHg}$, partial pressure of oxygen $140 \mathrm{mmHg}$, and bicarbonate 24 mmol/L. A discectomy and herniectomy with decompression of the thecal sac was performed, followed by surgical hemostasis .The patient's hemodynamic parameters remained stable during the procedure, and no vasopressors were required. Estimated blood loss was $300 \mathrm{ml}$, and the patient received a total of $1500 \mathrm{ml}$ of isotonic crystalloid with adequate urine output.

At the end of the surgery, anesthetic gases were discontinued, and the patient was awakened. Immediately following surgery, a neurological examination of the patient was performed to evaluate her motor and sensory functions, which were identical to the preoperative findings. Postoperative analgesia consisted of intravenous paracetamol $1 \mathrm{~g}$ every 8 hours and intravenous nefopam $100 \mathrm{mg}$ per 24 hours. Fetal ultrasound revealed a HR of 160 beats per minute. After discussion with the obstetrician, there was no indication forusing tocolysis. The patient was continuously monitored for another 24 hours in the intensive care unit, and the postoperative period remained uneventful. She was transferred to the rehabilitation department where she underwent physical therapy. On postoperative day 2 , she had slight sensation in both 
legs, and her muscle power showed a gradual improvement in the upper limbs $(3 / 5$ and $4 / 5$, respectively, in the right and left upper limbs). After 1 month, power had improved to grade 4 in her left leg and to grade 3 in her right leg, and she was able to move her arms freely.

\section{DISCUSSION}

Cervical disc herniation is 1 of the 23 specific disorders included in the CANS (Complaints of the Arm, Neck, and/or Shoulder) model [1] and the anesthetic management of $\mathrm{CDH}$ during prégnancy no case has been described in the littérature. General anesthesia is necessary for cervical localizations, and the difficulty concerns airway management of these patients. Masski et al., reported a case of spontaneous cervical epidural hematoma during pregnancy which presents the same anesthetic constraints as $\mathrm{CDH}$ who was under general anesthesia using a rapid sequence technique with cricoid pressure [2], in neutral position without a collar by a third person. Manual in-line stabilization (MILS) was used during direct laryngoscopy and intubation to minimize the risk of further cord compression. However, the MILS technique does not eliminate cervical spine movement [3], and it is known that MILS reduces mouth opening and leads to a poor laryngoscopic view [4].

In our patient, we used a different approach. We thought that conventional endotracheal intubation with direct laryngoscopy could have aggravated the injury and exposed the patient to a marked sympathetic response. Awake fiberoptic bronchoscopy was deemed most appropriate. The management of acute spinal cord decompression should also include measures to preserve spinal cord perfusion [5] and adequate uterine blood flow [6] by ensuring volume expansion, using anesthetics that respect the hemodynamic status and administering vasopressors if needed. Invasive BP monitoring with an indwelling arterial catheter is useful. Cardiac dysfunction secondary to loss of cardiac sympathetic tone is another reason for using invasive monitoring.

In the supine position the patient's head was maintained antero-Lateral positioning for an anterior surgical approch like a most of the cervical IDH in literature, but a larger portion of patients who underwent a posterior approach in prone position specially if the disc herniation is sequestrated with migration had improved recovery [7]. Bongetta affirm that the prone position is feasible during the second trimester of pregnancy provided an adequate frame is supplied and the lateral or three-quarters prone positioning may offer the safest option in the last stages of pregnancy [8].

Prone position is limited by the difficulty in monitoring the fetal HR and uterine activity. According to the American Congress of Obstetricians and
Gynecologists, the decision whether to use intraoperative fetal monitoring should be determined by a multidisciplinary team and based on each patient's unique circumstances and the surgery to be performed [9].

After a multidisciplinary team discussion, the supine position was selected for our patient to allow optimal surgical access.

\section{Conclusions}

This case report highlights the management of an uncommon medical condition in obstetrical patients. The potential for difficult airway management with the patient under general anesthesia is one of the major concerns. Anesthetic management should also include measures to maintain the mean arterial pressure to improve spinal cord perfusion, as well as special considerations regarding positioning and postoperative management.

\section{Abbreviations: \\ CDH: Cervical Disc Herniation; BP: Blood pressure; HR: Heart rate; MILS: Manual in-line stabilization; MRI: Magnetic resonance imaging}

\section{Acknowledgements: None.}

Funding: None.

Availability of Data and Materials: Relevant data and supporting materials will be made available on request.

\section{Authors' Contributions}

MS and MA drafted the manuscript under the supervision of MB. All authors participated in patient care. All authors critically revised the manuscript, and all authors read and approved the final manuscript.

Competing Interests: The authors declare that they have no competing interests.

Consent for Publication: Written informed consent was obtained from the patient for publication of this case report and any accompanying images.

Ethics approval and consent to participate : Not applicable.

\section{REFERENCES}

1. Huisstede BM, Miedema HS, Verhagen AP, Koes BW, Verhaar JA. Multidisciplinary consensus on terminology and classification of complaints of arm, neck and/or shoulder. Occupational and environmental medicine. 2006 Oct 16; 64:313-9.

2. Masski G, Housni B, Ibahiouin K, Miguil M. Spontaneous cervical epidural hematoma during pregnancy. Int J Obstet Anesth. 2004;13:103-6. 
3. Lennarson PJ, Smith DW, Sawin PD, Todd MM, Sato Y, Traynelis VC. Cervical spinal motion during intubation: efficacy of stabilization maneuvers in the setting of complete segmental instability. J Neurosurg. 2001;94(2 Suppl):265-70.

4. Heath KJ. The effect of laryngoscopy of different cervical spine immobilisation techniques. Anaesthesia. 1994;49:843-5.

5. Vale FL, Burns J, Jackson AB, Hadley MN. Combined medical and surgical treatment after acute spinal cord injury: results of a prospective pilot study to assess the merits of aggressive medical resuscitation and blood pressure management. J Neurosurg. 1997;87:239-46.

6. Braveman FR, Scavone BM, Wong CA, Santos AC. Obstetrical anesthesia. In: Barash PG, Cullen BF, Stoelting RK, Cahalan M, Stock MC, editors.
Clinical anesthesia. 6th ed. Philadelphia: Wolters Kluwer/Lippincott Williams \& Wilkins; 2009; 1137-67.

7. Gunasekaran A, de los Reyes NKM, Walters J, Kazemi N. Clinical Presentation, Diagnosis, and Surgica Treatment of Spontaneous Cervical Intradural Disc Herniations: A Review of the Literature, World Neurosurgery. 2017.

8. Bongetta D, Versace A, De Pirro A, Gemma M, Bernardo L, Cetin I, Savasi V, Assietti R. Positioning issues of spinal surgery during pregnancy. World Neurosurg. 2020 Jun;138:53-58.

9. Jo YY, Lee D, Chang YJ, Kwak HJ. Anesthetic management of a spontaneous spinal-epidural hematoma during pregnancy. Int J Obstet Anesth. 2012;21:185-8. 